\title{
A novel simple wideband common-mode suppression filter
}

\author{
Zhibin Zeng ${ }^{1,2 a)}$, Yiqi Zhuang ${ }^{1}$, Zengning Shen ${ }^{1}$, \\ Yindi $\mathrm{Yao}^{3}$, and Xin Xiang ${ }^{4}$ \\ ${ }^{1}$ School of Microelectronic, Xidian University, Xi'an 710071, China \\ ${ }^{2}$ Ministry of Education Key Lab. of Wide Band-Gap Semiconductor \\ Materials and Device, Xidian University, Xi'an 710071, China \\ ${ }^{3}$ Department of Telecommunication Engineering, \\ Xi'an University of Post and Telecommunications, Xi'an 710121 \\ ${ }^{4}$ Engineering College, Air Force Engineering University, Xi'an 710038, China \\ a)zbzeng@163.com
}

\begin{abstract}
A novel compact defected ground structure (DGS) common mode filter is proposed, which adopts only two C-shaped DGS and has only a small size of $7.5 \mathrm{~mm}$ by $10 \mathrm{~mm}$. It adopts two-pole structure to achieve wide stopband and adopts method of smooth transition at the junctions of DGS between the slit and the square to improve the depth of stopband. The filter provides over $15 \mathrm{~dB}$ common mode suppression from $3.7 \mathrm{GHz}$ to 9.8 $\mathrm{GHz}$, while the differential signals still keep good signal integrity. Simulated and measured results are well agreed.
\end{abstract}

Keywords: defected ground structure, common-mode filter, difference circuit, insertion loss

Classification: Microwave and millimeter wave devices, circuits, and systems

\section{References}

[1] S. Wu, C. Tsai, T. Wu and T. Itoh: IEEE Trans. Microw. Theory Tech. 57 (2009) 848. DOI:10.1109/TMTT.2009.2015087

[2] E. Pucci, E. Rajo-Iglesias and P.-S. Kildal: IEEE Microw. Wirel. Compon. Lett. 22 (2012) 129. DOI:10.1109/LMWC.2011.2182638

[3] F. de Paulis and A. Orlandi: IEEE Microw. Wirel. Compon. Lett. 19 (2009) 554. DOI:10.1109/LMWC.2009.2027062

[4] D. J. Woo, T. K. Lee, J. W. Lee, C. S. Pyo and W. K. Choi: IEEE Trans. Microw. Theory Tech. 54 (2006) 2840. DOI:10.1109/TMTT.2006.875450

[5] W. T. Liu, C. H. Tsai, T. W. Han and T. L. Wu: IEEE Microw. Wirel. Compon. Lett. 18 (2008) 248. DOI:10.1109/LMWC.2008.918883

[6] H. W. Liu, Z. F. Li, X. W. Sun and J. F. Mao: IEEE Microw. Wirel. Compon. Lett. 14 (2004) 180. DOI:10.1109/LMWC.2004.827097

[7] Y. J. Lu, S. Y. Chen and P. Hsu: IEEE Microw. Wirel. Compon. Lett. 22 (2012) 503. DOI:10.1109/LMWC.2012.2218279 


\section{Introduction}

Signal integrity is a critical issue in high-speed data communication. Differential signals have been widely used in high-speed digital circuits because they have advantages of low radiated emission and good immunity performance for common mode (CM) noise. However, because of unbalanced routing, patterned ground plane and clock skew, a significant amount of CM noises are easily generated in practical circuits [1]. They are responsible for electromagnetic interference (EMI) emissions problems which degrade the signal integrity of the high-speed circuit system.

One of the effective solutions for $\mathrm{CM}$ noise reduction is a $\mathrm{CM}$ filter. The conventional methods using discrete components are difficult to realize a large noise reduction effect in wide frequency range. Recent $\mathrm{CM}$ filter designs include electro-magnetic bandgap (EBG) filter [2, 3] and defected ground structure (DGS) filter $[1,4,5,6,7]$. The EBG filter is effective to reduce CM noise at the $\mathrm{GHz}$ band. However, it adopts periodic etched patches in the ground plane, which is difficult to provide enough space in portable embedded system, especially at microwave regime. Recently researchers focus on miniaturization and bandwidth enhancement for the DGS filter. It is found that the more DGS elements are used, the wider stopband is achieved. Reference [5] demonstrated a CM filter using periodic DGS can achieve a broad stopband from $3.3 \mathrm{GHz}$ to $5.7 \mathrm{GHz}$, of which the size is about $15 \mathrm{~mm}$ by $30 \mathrm{~mm}$. Reference [1] demonstrated that a CM DGS filter adopting two U-shaped and one H-shaped DGS cell can achieve a broad stopband from $3.6 \mathrm{GHz}$ to $9.1 \mathrm{GH}-\mathrm{z}$. The stop-band width and the size of the filter are $5.5 \mathrm{GHz}$ and $10 \mathrm{~mm}$ by $10 \mathrm{~mm}$ respectively. Because the compact device is important in the high density digital circuits, small size and wideband have been becoming one of the hottest topic in design of signal integrity.

In this letter, a new compact low-cost CM filter is proposed. The filter uses twopole filter structure to get wide stopband, and the depth of stopband is improved by smoothing the junction of DGS between the slit and the square to make the bottom of filter more flatness. With two optimized C-shaped DGS pattern on ground planes, it achieves a broad stopband width while it has a small area. The area of the proposed filter is only $75 \%$ of the CM filter in reference [1] while it has a wider stopband than that of the latter.

\section{Design and simulation}

The original structure of the proposed CM filter structure with two-pole DGS cells is shown in Fig. 1. Differential signals of coupled microstrip lines are routed on top of the patterned plane, and the DGS cells on ground planes are kept symmetrical to the central line of the two differential signal lines which is useful to avoid the excitation of CM noise. As shown in Fig. 1(a), on the bottom layer, each of the DGS dells is obtained by etching a slot connecting with two rectangular defected structures. Both of the two rectangular defected structures are the same. The structure dimensions are denoted as (w, d) for the coupled microstrip lines, (b, b1, s1, L1) for the left C-shaped DGS cell, and (b, b2, s2, L2) for the right C-shaped DGS cell. The distance between them is $\mathrm{L}_{3}$. 


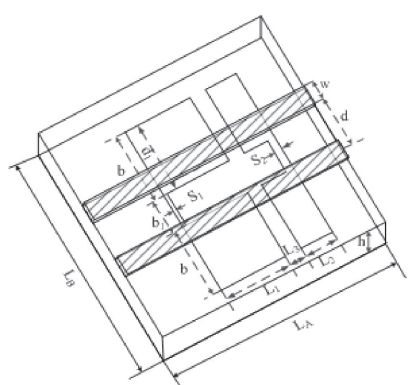

(a)

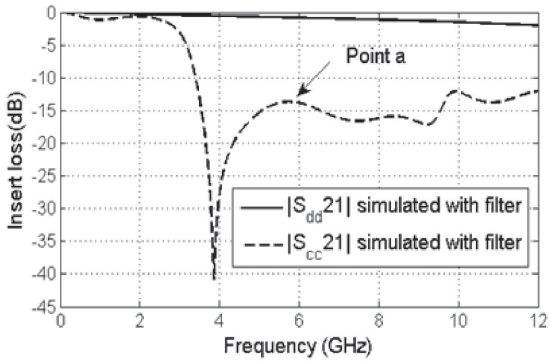

(b)

Fig. 1. Two-step common-mode DGS filter

(a) Schematic diagram of the proposed DGS filter

(b) Simulated common mode insertion loss

We built the band stop filter in a substrate whose relative permittivity is 4.4 and thickness is $0.8 \mathrm{~mm}$. And dimensional parameters of the filter are shown in Table I. The simulation result is given in Fig. 1(b). It is found the insertion loss for the CM in the wide stopband is approximately less than $15 \mathrm{~dB}$ within $3.6-9.5 \mathrm{GHz}$. The cutoff frequency is defined by $15 \mathrm{~dB}$, which is sufficient for solving the signal integrity and EMI issues in high-speed digital circuit applications [1, 5]. However, there exists a stub, named as point a, the insert loss Scc21 at point a is about $-13 \mathrm{~dB}$, which is over $-15 \mathrm{~dB}$ and needs to be further suppressed.

Table I. Simulation parameters used in original proposed commonmode filter

\begin{tabular}{lccccccccc}
\hline parameter & $\mathrm{w}$ & $\mathrm{d}$ & $\mathrm{b}$ & $\mathrm{b}_{1}$ & $\mathrm{~s}_{1}$ & $\mathrm{~L}_{1}$ & $\mathrm{~s}_{2}$ & $\mathrm{~L}_{2}$ & $\mathrm{~L}_{3}$ \\
\hline value $(\mathrm{mm})$ & 1.2 & 0.8 & 3.4 & 3.2 & 0.2 & 4.9 & 0.4 & 2 & 0.6 \\
\hline
\end{tabular}

\section{Improvement of CM filter}

In order to ensure the $\mathrm{CM}$ noise over the whole stopband is suppression depth more than $15 \mathrm{~dB}$, a measure named "smooth transition" is used to deal with the rightangle corners of A, B, C, D, as is shown in Fig. 2. Comparison of two methods is given here. A quarter circle with radius of $R_{i}(i=A, B, C, D)$ at inner corner is adopted in Method 1, as is shown in Fig. 2(b). A right angled triangle with length $\mathrm{LS}_{\mathrm{i}}$ of right angle side is adopted in method 2, as is shown in Fig. 2(c).

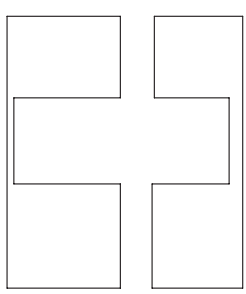

(a)

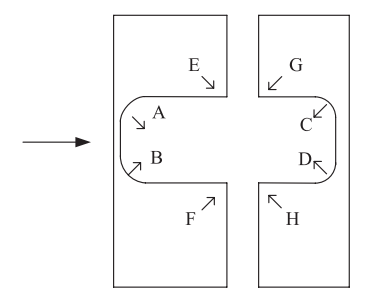

(b)

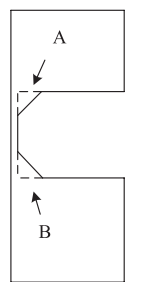

(c)

Fig. 2. Improved of two-step common-mode DGS filters. 
Fig. 3 shows the simulated results of CM DGS filter adopting various radius $R_{i}$ ( $\mathrm{i}=\mathrm{A}, \mathrm{B}, \mathrm{C}, \mathrm{D})$ or length $\mathrm{LS}_{\mathrm{i}}(\mathrm{i}=\mathrm{A}, \mathrm{B}, \mathrm{C}, \mathrm{D})$. When $\mathrm{LS}_{\mathrm{i}}=1.5 \mathrm{~mm}$, the CM noise suppression depth has a best effect. However, it has a highest low cutoff frequency of $-15 \mathrm{~dB}$. When $\mathrm{LS}_{\mathrm{i}}=0.5 \mathrm{~mm}$ or $\mathrm{R}_{\mathrm{i}}=1 \mathrm{~mm}$, the depth of CM noise suppression is not good. When $\mathrm{R}_{\mathrm{i}}=1.5 \mathrm{~mm}$ or $\mathrm{LS}_{\mathrm{i}}=1.0 \mathrm{~mm}$, the stopband width of CM filter is about $6.1 \mathrm{GHz}$ at the cutoff frequency of $-15 \mathrm{~dB}$. And the lower cutoff frequency of the CM filter is $3.71 \mathrm{GHz}$ when $\mathrm{R}_{\mathrm{i}}=1.5 \mathrm{~mm}$, and $3.78 \mathrm{GHz}$ when $\mathrm{LS}_{\mathrm{i}}=1.5 \mathrm{~mm}$. The lower cutoff frequency of the former is a litter less than that of the latter.

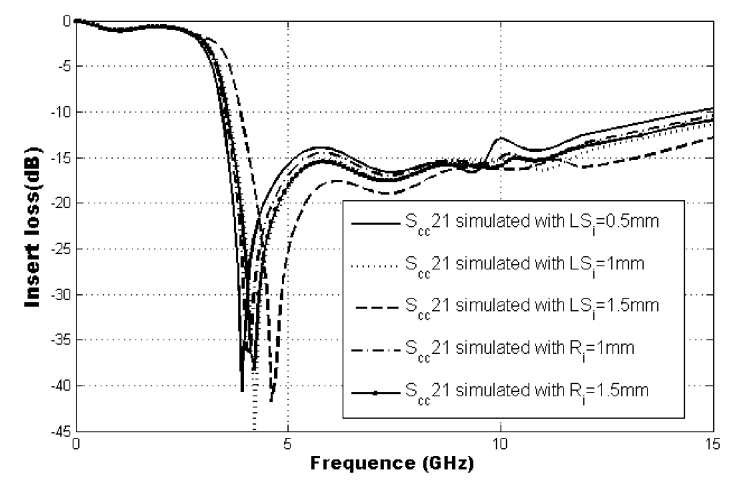

Fig. 3. Simulated insertion loss for common-mode filter adopting smooth transition with various $\mathrm{R}_{\mathrm{i}}$ and $\mathrm{LS}_{\mathrm{i}}$

In order to achieves a good flatness and depth in the CM DGS filter, "smooth transition" is also used at the corners of $\mathrm{E}, \mathrm{F}, \mathrm{G}, \mathrm{H}$. The improved CM filter has different radius at different corners. The radius of $R_{i}(i=A, B, C, D, E, F, G, H)$ is $1.5 \mathrm{~mm}, 1.5 \mathrm{~mm}, 0.7 \mathrm{~mm}, 0.7 \mathrm{~mm}, 0.8 \mathrm{~mm}, 0.8 \mathrm{~mm}, 0.6 \mathrm{~mm}$ and $0.6 \mathrm{~mm}$ respectively. Fig. 4 shows the simulated results before and after "smooth transition" being applied to the corners of E, F, G, H. The curves of both insert loss Scc21 are coincide under frequency of $5 \mathrm{GHz}$. But when frequency is higher than $5 \mathrm{GHz}$, the higher the frequency, the higher the suppression depth of the latter than that of the former.

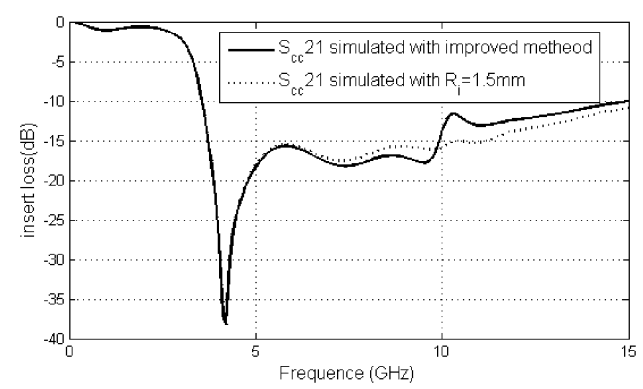

Fig. 4. Simulated insertion loss for common-mode filter before and after "smooth transition" at corners of E, F, G, H. 


\section{Evaluation of improved CM filter}

To validate the proposed DGB filter, it is fabricated on a FR4 substrate of dielectric constant 4.4. A photograph of the two-pole filter is presented in Fig. 5(a). The size of the board is $65 \mathrm{~mm}$ by $60 \mathrm{~mm}$. The total thickness of the board is $\mathrm{h}=0.8 \mathrm{~mm}$. Dimensional parameters of the filter are optimized and shown in Table II.

Table II. Geometric parameters used in test broad fabrication

\begin{tabular}{|l|c|c|c|c|c|c|c|c|c|}
\hline Parameter & $\mathrm{w}$ & $\mathrm{d}$ & $\mathrm{b}$ & $\mathrm{b}_{1}$ & $\mathrm{~s}_{1}$ & $\mathrm{~L}_{1}$ & $\mathrm{~s}_{2}$ & $\mathrm{~L}_{2}$ & $\mathrm{~L}_{3}$ \\
\hline Value $(\mathrm{mm})$ & 1.2 & 0.8 & 3.4 & 3.2 & 0.2 & 4.9 & 0.4 & 2 & 0.6 \\
\hline Parameter & $\mathrm{R}_{\mathrm{A}}$ & $\mathrm{R}_{\mathrm{B}}$ & $\mathrm{R}_{\mathrm{C}}$ & $\mathrm{R}_{\mathrm{D}}$ & $\mathrm{R}_{\mathrm{E}}$ & $\mathrm{R}_{\mathrm{F}}$ & $\mathrm{R}_{\mathrm{G}}$ & $\mathrm{R}_{\mathrm{H}}$ & \\
\hline Value $(\mathrm{mm})$ & 1.5 & 1.5 & 0.5 & 0.5 & 0.6 & 0.6 & 0.8 & 0.8 & \\
\hline
\end{tabular}

The measured insertion loss both for the differential mode and CM is shown in Fig. 5(b). And the full-wave simulated results are also presented for comparison. The fabricated filter is measured using vector network analyzer HP8363B. The dash dot line indicates the measured results and the solid line indicates the simulated results using HFSS 14.0. The wide stopband CM filter has a bandwidth of $3.7 \mathrm{GHz}$ to $9.8 \mathrm{GHz}$ at a cutoff frequency of $-15 \mathrm{~dB}$ for the simulation, and the differentialmode signals are almost intact observed from the differential transfer characteristics. The simulated and measured insertion losses of $\mathrm{CM}$ are almost the same such that their corresponding curves coincide with each other. The discrepancy between full-wave simulation and measurement may be caused by the length of transmission line, the usual connectors, manufacture tolerance, dispersive nature of the FR4 material and measurement error.

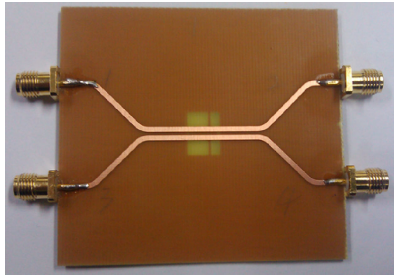

(a)

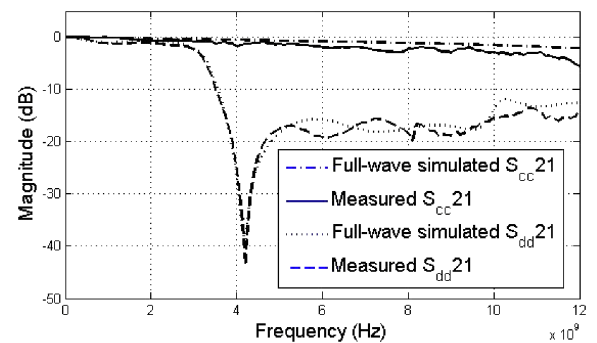

(b)

Fig. 5. Improved common-mode DGS filter

(a) Photo of the fabricated coplanar common-mode DGS filter (b) Simulated and measured insert loss of the common-mode DGS filter 
proposed structures shows a very good agreement with full-wave simulations. The proposed configuration can be easily implemented in real PCB design. The proposed structures may have wide applications in high speed digital design and microwave components.

\section{Acknowledgments}

The authors wish to acknowledge support from "National Science and Technology Major Project of the Ministry of Science and Technology of China (Grant No. 2012ZX03001018-001)" of China. and support from "the Fundamental Research Funds for the Central Universities (K5051125007)" of China. 